\title{
Metodología de evaluación con perspectiva de género (GEM) para telecentros: "una herramienta de aprendizaje para el cambio y el empoderamiento"
}

\author{
Evaluation Methodology with \\ Gender Perspective (GEM) for \\ Call Centers: A Learning Tool for \\ Change and Empowerment
}

Diana Marcela Escobar Aguirre (Colombia)

Universidad Autónoma de Occidente.

Grupo de Investigación en

Comunicación para el Desarrollo
Jorge Mauricio Escobar Sarria (Colombia)

Universidad Autónoma de Occidente.

Grupo de Investigación en

Comunicación para el Desarrollo

Olga Patricia Paz Martínez (Colombia)

Coordinadora proyecto GEM para Telecentros. Asociación Colombiana de Organizaciones no Gubernamentales para la Comunicación Vía Correo Electrónico (Colnodo)

\section{Resumen}

Gurumurthy (2004) afirma que la incorporación del enfoque de género en las tecnologías de la información
Abstract

Gurumurthy (2004) states that the incorporation of the gender approach in Information and Communication

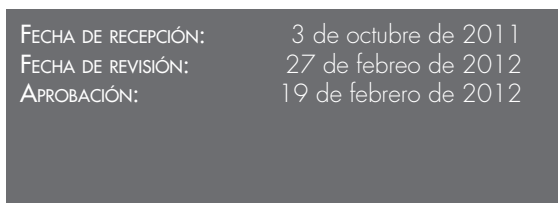

FECHA DE RECEPCIÓN: FECHA DE REVISIÓN: 27 de febreo de 2012 19 de febrero de 2012
Para citar este artículo / to Cite this article D Escobar Aguirre, D., Escobar Sarria, J. \& Paz Martínez, $\bigcirc$. Metodología de evaluación con perspectiva de género (GEM) para telecentros: una herramienta de aprendizaje para el cambio y el empoderamiento. Poliantea 8 (14), 163-188. 
y las comunicaciones (TIC) no tiene que ver solamente con un mayor uso de estas por parte de las mujeres, ya que implica una transformación del sistema donde se inscriben dichas tecnologías /contextos políticos, económicos, sociales, estrategias de promoción, formas de accesos y usos de las TIC, entre otros). En este sentido, los telecentros pueden llegar a ser espacios propicios para: 1) fomentar la participación de las mujeres en igualdad de condiciones con los hombres; 2) posibilitar la integración social; y 31 promover acciones o estrategias usando creativamente las TIC. Es así como la presente ponencia plantea, en primer lugar, una fundamentación conceptual de la relación existente entre TIC, desarrollo y género. En segundo lugar, reflexiona en torno al papel potencial de los telecentros como espacios para el empoderamiento y el cambio social. Por último, expone la adaptación para telecentros de la metodología de evaluación con perspectiva de género (Gender Methodology Evaluation, GEM), creada por el Programa de Apoyo a Redes de Mujeres (PARM) de la Asociación para el Progreso de las Comunicaciones (APC).

Palabras clave: género, tecnologías de la información y las comunicaciones (TIC), evaluación, cambio social, apropiación.
Technologies (ICTs) is not only related to their increasing use by women, but also to the transformation of the system where these technologies are included Ipolitical, economic and social contexts; promotional strategies, ways of access and uses of the ICT, among others). In this sense, call centers can become propitious spaces for: (1) fostering women participation with equality of conditions with men, (2) favoring the social integration and (3) promoting actions or strategies through the creative use of ICT. In this line, this paper proposes firstly a conceptual base of the existing relation among ICT, development, and gender. Secondly, it makes the reader reflect on the potential role of call centers as spaces for empowerment and social change. And lastly, the paper presents the adaptation of the Gender Methodology Evaluation (GEM) to call centers, which was created by the Support to Women's Network Program (PARM in Spanish) of the Association for Communication Progress (APC in Spanish).

Keywords: Gender, Information and Communication Technologies (ICT), evaluation, social change, appropriation. 


\title{
Metodología de evaluación con perspectiva de género (GEM) para telecentros: "una herramienta de aprendizaje para el cambio y el empoderamiento"
}

\author{
Diana Marcela Escobar Aguirre (Colombia) \\ Jorge Mauricio Escobar Sarria (Colombia) \\ Olga Patricia Paz Martínez (Colombia)
}

\section{Introducción}

La Gender Methodology Evaluation (GEM) o metodología de evaluación con perspectiva de género, en su traducción al español, diseñada por el Programa de Apoyo a Redes de Mujeres (PARM) de la Asociación para el Progreso de las Comunicaciones (APC), es una guía que incorpora un análisis de género en las evaluaciones de iniciativas que utilizan las tecnologías de la información y las comunicaciones (TIC) para el cambio social. Proporciona herramientas para determinar si las TIC apoyan procesos de cambio en relaciones de género, o si están promoviendo un cambio positivo individual, institucional, comunitario, o en los niveles sociales más amplios (APC, 2004).

La GEM para telecentros se desarrolla como una metodología complementaria a la guía GEM original, en la que se integra el enfoque y análisis de género desde y para los telecentros con el fin de potencializarlos como

\footnotetext{
Proyecto liderado por el Grupo de Investigación en Comunicación para el Desarrollo, de la Universidad Autónoma de Occidente (UAO), con la coordinación de la Asociación Colombiana de Organizaciones no Gubernamentales para la Comunicación Vía Correo Electrónico (Colnodo) y el acompañamiento técnico y financiero de la Asociación para el Progreso de las Comunicaciones (APC) y su Programa de Apoyo a Redes de Mujeres (PARM), junto al Ministerio de Tecnologías de la Información y las Comunicaciones, el Programa Compartel y la Red Nacional de Telecentros.
} 
espacios de integración comunitaria, de concertación ciudadana y de acceso equitativo a las TIC por parte de mujeres y hombres.

La adaptación de dicha metodología se presenta en el proyecto denominado "Implementación de la herramienta GEM en los telecentros Compartel de Villapaz y Santa Elena, localizados al suroccidente de Colombia", que buscó sensibilizar tanto a mujeres como a hombres sobre la función que cumplen en la vida doméstica, comunitaria o social, y las relaciones de poder que establecen entre ambos sexos; así como también, el análisis de sus problemas y la búsqueda de medidas adecuadas para resolverlos, especialmente mediante usos, prácticas y procesos de apropiación social de las TIC.

En otras palabras, el proceso se planteaba pasar de la asistencia social al acceso, de manera que las mujeres y los hombres lograran reconocer y analizar sus problemas y tomar medidas para resolverlos por medio del uso de las TIC; un tránsito de la sensibilización a la movilización de las mujeres a partir de un proceso de formación que les permita analizar sus problemas y buscar alternativas para atenderlos, al identificar necesidades específicas con respecto a las TIC y responder, entre otras, las siguientes preguntas:

- ¿El telecentro ofrece información especialmente para los intereses de las mujeres?

- ¿Cómo desde la planeación del telecentro se están resolviendo esas necesidades?

- ¿El telecentro promociona y favorece el uso estratégico de las TIC por parte de las mujeres?

- ¿El telecentro ofrece oportunidades de capacitación y formación en el uso de TIC por parte de las mujeres?

- ¿El telecentro ofrece cursos y oportunidades de formación y educación virtual en los que puedan participar mujeres y hombres para mejorar su cualificación y competencias, especialmente con miras a mejorar su nivel de ingresos?

- ¿Cuáles son los prejuicios que tienen mujeres y hombres sobre los servicios que ofrecen el telecentro y las TIC?

- ¿La persona que administra el telecentro está sensibilizada en la perspectiva de género?

En suma, el empoderamiento de las mujeres en la sociedad de la 
información con miras a un cambio social y un enfoque de desarrollo equitativo requiere de un constante análisis a las maneras en que las relaciones de género, como proceso cultural dinámico, son negociadas y rebatidas en lo que se refiere al entorno de la tecnología. Históricamente la tecnología ha sido un campo masculino; por tanto, sugiere que la apropiación de esta por las mujeres sea en sí un proyecto político que derive en acciones de investigación, intervención y reflexión con perspectiva de género (Gurumurthy, 2004).

La APC (2006) declara:

Un acceso asequible rápido y fácil a Internet puede ayudar a generar sociedades más igualitarias. Puede servir para fortalecer los servicios de educación y salud, el desarrollo económico local, la participación pública, el acceso a la información, la buena gobernanza y la erradicación de la pobreza. Pero no habría que dar por sentado que la innovación tecnológica genera un beneficio automático. Las organizaciones de la sociedad civil (OSC), los Gobiernos y los entes reguladores deberían ser conscientes del potencial de Internet para reforzar las desigualdades existentes.

\section{Fundamentación conceptual: una mirada a la relación existente entre TIC, desarrollo y género}

Según el Programa de las Naciones Unidas para el Desarrollo (PNUD, 2007), los estudios de la mujer que se iniciaron en los años ochenta propiciaron una delimitación de las políticas de desarrollo que se concibieron desde mediados del siglo XX, las cuales han ido evolucionando desde un enfoque "asistencialista" hasta llegar a la concepción de políticas de igualdad de género que incluyen a los Estados por medio del ejercicio y la proclamación de sus políticas públicas.

Dentro de lo anterior, existen dos tendencias reconocidas en los modelos y estrategias globales de desarrollo que abordan el tema de las mujeres: "Mujeres en el Desarrollo (MED)" y "Género en el Desarrollo (GED)”. El MED tiene por objetivo la integración de las mujeres a las estrategias de desarrollo desde una mirada funcional, en la que se da preponderancia al papel reproductivo de estas y la subordinación o exclusión que en el mercado laboral esto implica. De ese modo, considera a las mujeres de manera aislada, busca soluciones parciales y señala sus necesidades mediante intervenciones puntuales en componentes específicos de proyectos (De la Cruz, 1999). De la 
segunda tendencia (GED), De la Cruz (1999) plantea principalmente:

- Promueve la eficiencia y la identificación de las oportunidades para mejorar la redistribución de género y la equidad en las políticas, proyectos $y$ programas de desarrollo.

- Implica que las necesidades de las mujeres dejen de ser analizadas aisladamente para ser parte integrante de un análisis de las relaciones de género en los hogares, en la comunidad y en las instituciones.

- Busca el "empoderamiento" de las mujeres y de los colectivos en desventaja, e incluye la satisfacción de las necesidades prácticas de género para asegurar la alimentación, vivienda, agua y autosuficiencia económica.

Ahora bien, las desigualdades basadas en el sexo han sido un rasgo persistente de todas las sociedades, resultado de relaciones de poder, normas y prácticas construidas socialmente. Por tanto, cabe afirmar que cualquier alternativa o propuesta de desarrollo humano tiene la obligación de considerar la perspectiva de género; y se debe comprender a partir de las declaraciones de la IV Conferencia Mundial sobre la Mujer (Beijing, 1995) que el género se refiere a los papeles sociales construidos para la mujer y el hombre asentados con base en su sexo, dependientes de un contexto socioeconómico, político y cultural y afectados por otros factores como la edad, la clase, la raza y la etnia.

En este orden de ideas, el desarrollo está intrínsecamente relacionado con las cosas que las personas pueden realmente hacer o ser, y por tanto, con las capacidades que disponen para hacerlo; es decir, las oportunidades para elegir y llevar una u otra clase de vida. En este sentido, el desarrollo es el camino a una "libertad mayor", lo cual significaría que una sociedad desarrollada es sinónimo de una sociedad más libre. Así, el desarrollo comprende posibilidades elementales de acceso a espacios o entornos tales como el educativo, laboral, de la salud, de acceso a la información, la participación política o la seguridad colectiva (Córdoba, 2007).

Casttels (2008) asegura además que el desarrollo está determinado por la capacidad de establecer una interacción sinérgica entre la innovación tecnológica y los valores 
humanos, con base en que las diversas esferas en las que se moviliza toda actividad humana dependen tanto del poder de la información como de una secuencia de innovación tecnológica que en el siglo actual cada vez cobra mayor relevancia. Si bien la tecnología per se no resuelve los problemas sociales, su disponibilidad y uso sí resultan ser un prerrequisito para el desarrollo económico y social del mundo actual.

Las cumbres mundiales sobre la sociedad de la información (CMSI) (Ginebra, 2003; Túnez, 2005), declararon el acceso y uso de las TIC como vitales para el empoderamiento de las mujeres:

Afirmamos que el desarrollo de las TIC brinda ingentes oportunidades a las mujeres, las cuales deben ser parte integrante y participantes clave de la Sociedad de la Información. Nos comprometemos a garantizar que la Sociedad de la Información fomente la potenciación de las mujeres y su plena participación, en pie de igualdad, en todas las esferas de la sociedad y en todos los procesos de adopción de decisiones. A dicho efecto, debemos integrar una perspectiva de igualdad de género y utilizar las TIC como un instrumento para conseguir este objetivo (apartado A, numeral 12).

Dentro de lo anterior, la relación existente entre TIC y desarrollo (al considerar la perspectiva de género), puede ser comprendida a partir de dos escenarios: 1) las TIC han dado lugar a un nuevo espacio público global, donde emergen las nuevas formas que cobran los derechos de primera, segunda y tercera generación; y 2) la concreción de los derechos a la información, la comunicación y el ciberespacio, en la sociedad de la información, no solo se convierten en derechos humanos fundamentales en sí, sino también en una condición para el ejercicio y la defensa de los demás derechos (Del Río, 2009).

Es así como desde la mirada de un proceso de democratización se ha demostrado que el acceso y el uso estratégico de las TIC tienen el potencial para promover el desarrollo económico, la reducción de la pobreza y la democracia -que incluye libertad de expresión, libre flujo de información y defensa de los derechos humanos- (Asociación para el Progreso de las Comunicaciones, [APC], 2009). Por tanto, la transversalidad de las TIC en las diversas esferas del desarrollo humano demanda con urgencia su inclusión entre de los derechos de la información y la comunicación, y considera el ciberespacio como 
un nuevo escenario en el que estos pueden ejercerse o vulnerarse (Del Río, 2009).

A lo anterior debe sumarse la falta de estadísticas o datos desagregados por sexo e indicadores de género en las TIC, que en muchos países no existen o no se caracterizan con facilidad. El análisis de cómo los hombres y las mujeres hacen uso de las TIC y el impacto que ellas generan resulta un requisito necesario para el logro de una sociedad de la información a nivel mundial, ya que sin dicha información más del cincuenta por ciento de la población mundial fácilmente se pasa por alto (Hafkin \& Huyer, 2007).

Ahora bien, en lo que respecta a la denominada "brecha digital”, para Teltscher (2002) resulta mayor en países donde las mujeres tienen menos acceso a la educación que los hombres (como es el caso en muchos países pobres o catalogados como "en vía de desarrollo"), y en aquellos que por razones culturales o religiosas discriminan a la mujer. En ambos casos las mujeres siguen siendo una pequeña minoría en Internet.

Hasta el momento la relación existente entre TIC, desarrollo y género se ha procurado establecer grosso modo, y se destacan algunas de sus principales ventajas o avances, así como las dificultades y desafíos que aún persisten o están por trabajar. En este orden de ideas vale la pena destacar la reflexión de MartínBarbero (2004) en torno al concepto de sociedad de la información, en el que las múltiples "mutaciones societales" que experimentan, por primera vez, tanto la idea como la realidad del mundo, no pueden ser vistas tan solo desde lo que sucede con la población "conectada", sino, tanto o más, con la "desconectada". "La mirada crítica nos advierte certeramente de los riesgos que entraña el actual desarrollo tecnológico en sus complicidades con las lógicas del mercado y los procesos de agravamiento de la exclusión social" (Martín-Barbero, 2004, p. 10).

Es así como la denominada "brecha digital" arriba destacada, no es más que una brecha social, pues no remite a un mero efecto de la tecnología digital, sino que trasciende a la organización de una sociedad que en la mayoría de los casos impide, no solo acceder, sino apropiarse de manera física, económica y mental de dichas tecnologías (Martín-Barbero, 2004).

Kofi Annan, secretario de las Naciones Unidas en el 2003, al 
destacar la relación existente entre género y TIC deriva la denominada brecha digital en tres: 1) brecha tecnológica, o de grandes desigualdades en infraestructura; 2) brecha de contenido, en la que una gran cantidad de información basada en el ciberespacio simplemente no es relevante para las necesidades reales de la gente y casi el setenta por ciento de los sitios de Internet del mundo están en inglés, lo que a veces deja por fuera las voces $y$ opiniones locales; y (3) brecha de género, en la que las mujeres y las niñas gozan de menos acceso que los hombres y los niños a la tecnología de la información.

Hoy por hoy se hace imposible ignorar que las TIC resultan esenciales para el desarrollo tanto económico como para el bienestar material; de acuerdo con Castells (2008), estas condicionan el poder, el conocimiento y la creatividad, a la vez que propician un círculo virtuoso de desarrollo o una espiral descendente del subdesarrollo. En tanto, el manejo y acceso a la información que de manera permanente circula a través de las TIC, así como su adecuación y la capacidad que tengan los individuos de generar conocimiento al entrar en contacto con dicha información, resulta un requisito fundamental para la ampliación de oportunidades y, por ende, para que las personas logren un nivel de desarrollo que les propicie una vida digna y duradera (Rueda, 2005).

Los denominados telecentros surgen así como una estrategia para que poblaciones en condiciones de vulnerabilidad puedan "conectarse". Según el PNUD (2003), dicho fenómeno es uno de los más extendidos a nivel internacional en la esfera de las TIC y consiste en la instalación de centros públicos con diversas configuraciones que permiten el acceso comunitario a las TIC y al conocimiento que dicha tecnología facilita. Sus antecedentes se encuentran en países desarrollados como Canadá, Estados Unidos, Australia o Suecia (con términos como telecottages, community technology centers, telecabinas o telecentros), donde desde principios de los noventa se encontraban estos centros para facilitar el acceso local a las TIC cuando las conexiones personales a Internet y la posesión individual de ordenadores todavía eran muy limitadas. 


\section{Telecentros como espacios propicios para fortalecer procesos de empoderamiento y cambio social}

Sabanes (2004) afirma que con el objetivo de generalizar el acceso a las TIC y a las fuentes de conocimiento, información y comunicación se crearon los telecentros barriales o comunitarios, que, con la administración de una organización vecinal o local, brindan conectividad y acceso a las TIC en zonas urbanas periféricas, barrios marginados o áreas rurales. Dichos telecentros constan por lo general de una sala con computadoras conectadas a Internet y se ofrecen allí capacitaciones en TIC, conexión y la posibilidad de aplicar las TIC para el desarrollo comunitario. Brindan sus servicios a bajos costos y tienen en cuenta las posibilidades reales de la población circundante.

En lo que respecta a la reducción de la denominada brecha digital resulta fundamental, por tanto, la extensión de la infraestructura, en particular comunicaciones inalámbricas y por satélite a las áreas rurales y periurbanas, así como la promoción de iniciativas encaminadas a aumentar el acceso a partir de instalaciones de uso común tales como telecentros, tiendas con teléfonos para la comunidad y otros lugares públicos convenientes y accesibles tanto para las mujeres como para los hombres (Hafkin \& Taggart, 2009).

Los proyectos exitosos en países en desarrollo muestran que los beneficios económicos de las TIC dependen del desarrollo de capacidades por parte de sus usuarios/ as, lo cual deja de lado el uso operativo de las TIC y promueve la promoción y desarrollo de contenidos relevantes para la localidad; el establecimiento de empresas (de TIC y ayudadas por las TIC); usar Internet para comunicación y articulación a través de redes; fomentar la promoción y defensa pública; forjar vínculos con instituciones locales e integrar las actividades económicas de poblaciones más vulnerables en los mercados locales y globales (Gurumurthy, 2004).

No obstante, aún persiste la distribución desigual de la información y la tecnología (IT) en las sociedades y en el mundo está resultando en una "división digital" entre quienes tienen acceso a los recursos de la información y quienes no lo tienen; y es así como la mayoría de las mujeres en los países en desarrollo 
se encuentran en el fondo de dicha división, más apartadas de la era de la información que los hombres; aun cuando comparten condiciones de pobreza, los niveles más bajos de alfabetización y educación los reflejan las mujeres, así como las actitudes negativas hacia los resultados escolásticos de las muchachas en las ciencias y las matemáticas, todo lo cual contribuye a una división digital en función del género (Hafkin y Taggart, 2009).

En suma, así como el acceso a las TIC por sí solo no reducirá la pobreza, las TIC por sí mismas no resolverán las inequidades de género existentes. Sin embargo, resultará fundamental el fomentar el acceso, aprendizaje, uso y apropiación social de las TIC en procura de cambiar los papeles tradicionales y las relaciones de poder preestablecidas entre hombres y mujeres (Finquelievich \& Lago, 2004).

Finquelievich y Lago (2004), en dos investigaciones realizadas entre agosto de 2002 y julio de 2003: "TIC y desarrollo local. El caso de la región norte del Área Metropolitana de Buenos Aires" y "TIC y reducción de la pobreza en América Latina y el Caribe (ALC)", identifican muy pocas iniciativas que aún se dirigen a la formación de mujeres en herramientas de TIC, o a su empleabilidad en trabajos que impliquen el uso de estas tecnologías. Los programas y planes existentes ni siquiera denuncian la inequidad en el acceso y formación a las TIC entre los géneros y rara vez incorporan componentes importantes favorables a las mujeres o examinan la forma en que sus componentes beneficiarán a las mujeres pobres. Para el caso del género, más grave que en otros sectores, no existe prácticamente articulación entre planes sociales y planes relativos a TIC dirigidos a las mujeres.

\section{Caso GEM (Gender Methodology Evaluation) para telecentros Gender Methodology Evaluation (GEM)}

La GEM, creada por el Programa de Apoyo a Redes de Mujeres (PARM) de la Asociación para el Progreso de las Comunicaciones (APC), es una metodología que integra el análisis de género en la evaluación de iniciativas que usan TIC para el cambio social. Provee herramientas para constatar si las TIC realmente mejoran la condición de las mujeres y las relaciones de género, así como promueven cambios positivos en 
los niveles individual, institucional, comunitario y social (Sabanes, 2004).

Según la PARM (2004), la guía brinda la posibilidad de que la organización o grupo coordinador arme el proceso de evaluación del proyecto elegido de acuerdo con sus objetivos, limitaciones y posibilidades en los que se tengan presentes los siguientes aspectos:

- Teórico: un marco analítico con perspectiva de género, conceptos y principios fundamentales para la evaluación.

- Práctico: enfoques metodológicos para conducir la evaluación.

- Acción: una evaluación vinculada a la acción hace énfasis en la utilización de lo que se aprende. La clave está en conectar la información con el cambio propuesto.

- Aprendizaje a partir de la acción: observar y reflexionar sobre las actividades del proyecto y el contexto en el cual se llevan a cabo.

- Sensibilidad de género: comprender las disparidades de género en el acceso y control de los recursos es un componente fundamental para realizar una evaluación con perspectiva de género.

- Cambio individual y social: un proceso de evaluación debe analizar la iniciativa de TIC y el contexto social más amplio.

- Participativo: el proceso de evaluación debe involucrar a todos/as los/as participantes y sus objetivos ser compartidos de manera participativa para asegurar la transparencia.

Dentro de lo anterior, la implementación de la GEM requiere el desarrollo de tres etapas: 1) integración del análisis de género en la evaluación; 2) obtención o recolección de la información con la utilización de indicadores de género y TIC; 3 ) puesta en marcha de los resultados de la evaluación.

\section{Antecedentes del caso y contextualización de su aplicación}

La GEM para telecentros recopila el proceso de investigación desarrollado en el proyecto "Implementación de la herramienta GEM en los telecentros Compartel de Villapaz y Santa Elena, localizados al suroccidente de Colombia", el cual se dio luego de que ambos 
telecentros hubiesen sido beneficiarios del proyecto "Gestión del conocimiento e intercambio de experiencias entre telecentros comunitarios y telecentros Compartel en Colombia", realizado entre septiembre de 2006 y diciembre de 2007, coordinado por Colnodo en alianza con la Universidad Autónoma de Occidente (UAO), el aval del Programa Compartel y el apoyo técnico y financiero de telecentre.org.

Dicho proyecto tenía entre sus objetivos principales:

- El desarrollo o puesta en marcha de metodologías, actividades y recursos que dieran lugar a procesos de apropiación social de las TIC, que a su vez permitieran mejorar las condiciones y calidad de vida de las personas o comunidades cercanas al telecentro.

- La producción de contenidos y la difusión de estos entre telecentros en Colombia.

- El fortalecimiento de la Red Nacional de Telecentros (www. Telecentros.org.co).

En el momento de realizar el proceso de valoración general de los resultados y lecciones aprendidas se identificaron necesidades específicas de parte de las mujeres que participaron en el proceso con respecto a los usos y prácticas de apropiación social de las TIC que se daban en el telecentro y su entorno más cercano. Lo anterior propició que, a partir de la lectura, análisis e interpretación de la guía GEM original, se desarrollara el proyecto piloto de implementación de la metodología para el caso de los telecentros, con el objetivo principal de permitir el análisis de usos y prácticas de las TIC desde una perspectiva de género.

Las comunidades seleccionadas para el proceso fueron: 1) Villapaz, que tiene un promedio de 5000 habitantes, principalmente de etnia afrodescendiente; y 2) Santa Elena, con aproximadamente 12000 habitantes, en su mayoría blancos o mestizos.

La economía del primer corregimiento consiste básicamente en la siembra de frutales y otras especies, en la cría de bovinos o parisinos en algunas haciendas, y en general la comunidad se dedica a las labores de agricultura que demanda el campo. Mientras que Santa Elena, el segundo corregimiento, se caracteriza por sus diversos atractivos turísticos, que terminan siendo 
uno de los principales motores económicos de la zona, entre ellos: la hacienda El Paraíso; el cementerio Central Católico; la "choza de Chuchú", donde se puede encontrar gran diversidad de materiales y accesorios de las diferentes épocas; el Museo de la Caña de Azúcar y una gran cantidad de fincas campestres.

En cuanto a los participantes y según los resultados de una encuesta aplicada en noviembre de 2009 para caracterizar los/las participantes en el proceso de implementación de la metodología, fueron en su mayoría mujeres, pese a que la convocatoria se abrió tanto para hombres como para mujeres.

En Villapaz la encuesta se aplicó a 31 personas, de estas el $87 \%$ fueron mujeres, es decir, que de 31 personas encuestadas, 27 fueron mujeres, mientras que en Santa Elena dicho porcentaje fue del 69\%: de 29 encuestados, 20 fueron mujeres.

Asimismo, se evidenció que ambos telecentros son visitados por personas entre los 14 y 26 años de edad, o 27 y 35 años de edad, predominando en un 39\% dichos rangos. De las 29 personas encuestadas en
Santa Elena y las 31 personas encuestadas en Villapaz, ambos telecentros tienen en su mayoría usuarios jóvenes; adicional a esto, gran parte de los caracterizados son solteros y sin hijos.

En cuanto al grado de escolaridad, en ambos telecentros (Villa Paz y Santa Elena) la mitad de los/as usuarios/as aproximadamente, cursaron el bachillerato completo; los demás, incompleto. Esto significa que en Villapaz el $45 \%$, equivalente a catorce personas, son bachilleres graduados y el 39\%, equivalente a trece personas, no lo son. En Santa Elena sucede algo similar: el $41 \%$, equivalente a doce personas, cursaron el bachillerato completo, y el $31 \%$, equivalente a nueve personas, no lo han terminado aún.

Por último, respecto a los usos del telecentro, los encuestados de ambos telecentros le asignaron una mayor calificación al uso educativo; y referente a las motivaciones que tienen para visitar el telecentro, la principal resultó ser la de mejorar su educación y encontrar oportunidades laborales. 


\section{Aspectos relevantes de la implementación de la GEM para telecentros Objetivos general}

Aumentar la participación de las mujeres, concientizarlas sobre las problemáticas de su entorno y lograr la movilización de acciones y estrategias con el uso creativo de las TIC en el telecentro.

\section{Objetivos específicos}

- Conocer los usos que mujeres y hombres usuarias/os del telecentro hacen de las TIC, y especialmente reconocer usos diferenciados por género.

- Conocer si el uso de las TIC ha ofrecido ventajas y permitido cambios en la vida de mujeres y hombres del entorno del telecentro.

- Involucrar a la persona que administra el telecentro en la promoción de las TIC con perspectiva de género.

- Fortalecer a las mujeres de la comunidad, concientizarlas sobre su función y motivarlas para aumentar su participación en la atención de problemáticas sociales con el uso estratégico de las TIC.

- Construir buenas prácticas para lograr que mujeres y hombres usen y se apropien de las TIC desde los telecentros en temas directamente relacionados con el mejoramiento de sus condiciones de vida.

- Aumentar el impacto de los telecentros en el desarrollo social y la calidad de vida del entorno, de manera equitativa, para hombres y mujeres.

\section{Cuestiones de género propuestas para la implementación de la GEM en los telecentros}

A partir de las problemáticas de género identificadas se establecieron preguntas que orientaran la implementación de la metodología de evaluación, así como una serie de indicadores de orden cualitativo y cuantitativo, para finalizar con el proceso de recolección y análisis de la información. Se presentan así los siguientes cuadros:

\section{Sistematización de la}

\section{adaptación de la metodología GEM para telecentros}

Para Ruiz (2001), la sistematización es la realización de un trabajo consciente (del equipo investigador) por recopilar los significados de la acción y sus efectos, realizar lecturas organizadas de las experiencias, teorización y cuestionamientos 


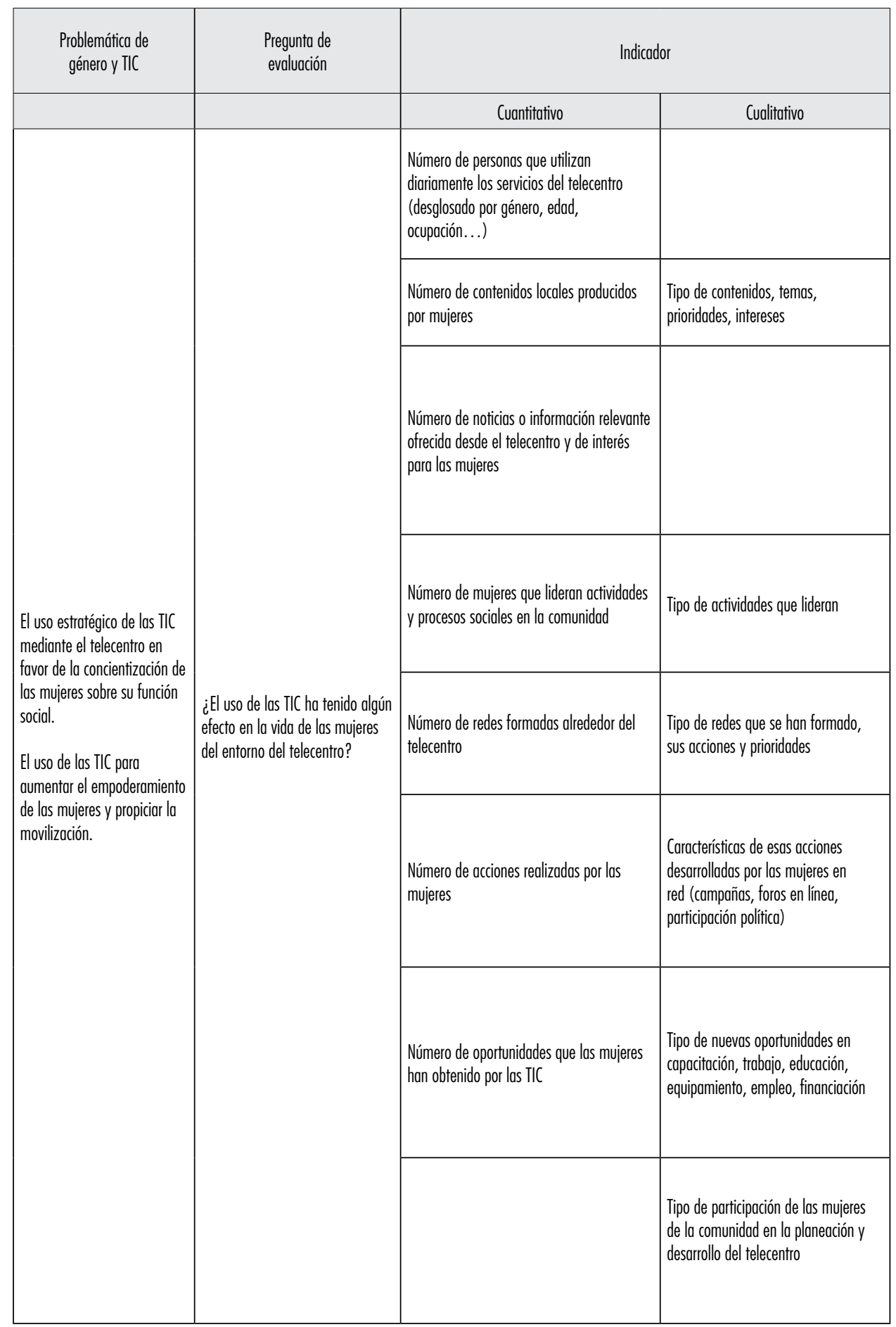




\begin{tabular}{|c|c|c|c|}
\hline \multirow{2}{*}{$\begin{array}{c}\text { Problemática de } \\
\text { género y TIC }\end{array}$} & \multirow[t]{2}{*}{ Pregunta de evaluación } & \multicolumn{2}{|r|}{ Indicador } \\
\hline & & Cuantitativo & Cualitativo \\
\hline \multirow{5}{*}{$\begin{array}{l}\text { Impacto en las mujeres de } \\
\text { las capacitaciones en uso de } \\
\text { TIC que ofrece el telecentro. } \\
\text { Nuevas capacidades de } \\
\text { los hombres y las mujeres } \\
\text { que han participado en los } \\
\text { talleres sobre TIC. }\end{array}$} & \multirow{10}{*}{$\begin{array}{l}\text { ¿Qué aspectos } \\
\text { deben considerarse } \\
\text { en la capacitación } \\
\text { para favorecer la } \\
\text { participación de las } \\
\text { mujeres? }\end{array}$} & $\begin{array}{l}\text { Número de personas que participan } \\
\text { en capacitaciones sobre el uso de } \\
\text { TIC, desagregado por género, edad y } \\
\text { ocupación. }\end{array}$ & $\begin{array}{l}\text { Tipo de capacidades adquiridas (correo } \\
\text { electrónico, navegación por Internet, herramientas } \\
\text { colaborativas, participación en discusiones en } \\
\text { línea, listas, producción de contenidos, sitio web } \\
\text { o bitácora, etc.) }\end{array}$ \\
\hline & & & $\begin{array}{l}\text { Características de metodologías de capacitación } \\
\text { diseñadas desde el telecentro (revisar si hay } \\
\text { metodologías diferenciadas para hombres, } \\
\text { mujeres, niños/as, jóvenes, adultos/as mayores) }\end{array}$ \\
\hline & & & $\begin{array}{l}\text { Horarios, costos, actividades y temas propuestos } \\
\text { para la capacitación }\end{array}$ \\
\hline & & $\begin{array}{l}\text { Número de medios locales por medio } \\
\text { de los cuales se han difundido la } \\
\text { capacitación y los servicios del telecentro }\end{array}$ & $\begin{array}{l}\text { Tipo de mensajes de promoción de la capacitación } \\
\text { y de los servicios del telecentro }\end{array}$ \\
\hline & & $\begin{array}{l}\text { Número de preguntas que las mujeres } \\
\text { realizan durante la capacitación }\end{array}$ & $\begin{array}{l}\text { Cuestiones que preocupan a las mujeres en su } \\
\text { relación con la tecnología } \\
\text { Tipo de conocimientos que desean adquirir o tener } \\
\text { acceso y también producir con el uso de las TIC }\end{array}$ \\
\hline \multirow{5}{*}{$\begin{array}{l}\text { Efectos de la capacitación } \\
\text { en la autoestima de las } \\
\text { mujeres. }\end{array}$} & & $\begin{array}{l}\text { Número de mujeres que han llegado a } \\
\text { las capacitaciones animadas por otras } \\
\text { mujeres que se han capacitado }\end{array}$ & \\
\hline & & $\begin{array}{l}\text { Número de oportunidades que han tenido } \\
\text { las mujeres luego de las capacitaciones }\end{array}$ & $\begin{array}{l}\text { Tipo de oportunidades que han tenido las mujeres } \\
\text { luego de participar en la capacitación en TIC. }\end{array}$ \\
\hline & & $\begin{array}{l}\text { Número de capacitaciones ofrecidas por la } \\
\text { persona administradora del telecentro }\end{array}$ & \\
\hline & & & $\begin{array}{l}\text { Evaluación y valoración de las mujeres sobre las } \\
\text { capacitaciones. }\end{array}$ \\
\hline & & & $\begin{array}{l}\text { Fortalecimiento del aumento de autoestima, } \\
\text { mayor confianza en sí mismas. }\end{array}$ \\
\hline
\end{tabular}


contextualizados de la praxis social, con el propósito de comunicar el conocimiento producido. Como esfuerzo de sistematización y con la finalidad de escalar a un nivel nacional la metodología GEM adaptada en los telecentros de Villapaz y Santa Elena, se desarrolló un ejercicio de reorganización de datos, entrevistas, cifras, herramientas diseñadas, entre otras, para consolidar un producto multimedia que permitiera compilar y dar cuenta a los diversos actores involucrados en el proyecto de los resultados, hallazgos, aprendizajes o experiencias significativas que se han obtenido.

No obstante, la sistematización es un proceso que con frecuencia se dilata y no se concreta en los proyectos que combinan la investigación teórica con la intervención, puesto que ni los conocimientos o la fundamentación teórico-conceptual en la que se sustenta la intervención inicial, ni el nuevo conocimiento que se genera durante y posterior a dicha intervención, propician las condiciones para estructurar un proceso de saber (conocimiento ordenado y, por tanto, transmisible). Justamente la sistematización trata, por ello, de ser un proceso que oriente a los profesionales, así que brinda orden y rigor al conocimiento que encuentran en su práctica (Bernechea, 1994).

Los telecentros como espacios de la comunidad y para la comunidad puede ser considerada la premisa de la adaptación de la metodología GEM, puesto que por medio de su estructuración, mensajes y productos los promueve como lugares propicios para la formación en el uso de las TIC, la articulación de alianzas, la atención a problemáticas del entorno local y la integración social de hombres y mujeres en igualdad de condiciones.

Teniendo en cuenta lo anterior, el proceso de sistematización para un formato multimedial se dividió en cinco fases principales, las cuales surgieron de las experiencias, recomendaciones o percepciones de los/ las participantes, lecciones aprendidas, sugerencias de colegas y autoevaluaciones realizadas por los integrantes del equipo de trabajo durante el proyecto.

En este punto se hace necesario recordar que la metodología o cartilla GEM original desarrolla tres fases: 1) integración de análisis de género; 2) recolección de información con la utilización de indicadores de género; 3) TIC y aplicación 
de los resultados de la evaluación. En GEM para telecentros, como se dijo, dichas fases se ampliaron a cinco y con nombres simplificados de modo que permitiesen una mayor interpretación del proceso como tal.

\section{Fase l: Sensibilizar}

Busca confrontar a los/las participantes mediante el análisis de sus vivencias cotidianas, de sus problemáticas, de sus creencias y sus paradigmas. Con la sensibilización se explora e indaga en los participantes la manera como asumen sus papeles sociales, cómo establecen sus relaciones de poder y cómo participan en la comunidad.

La estrategia es la puesta en marcha de actividades, talleres o trabajos lúdicos que invitan a confrontar el significado de ser hombre o ser mujer. Adicional a ello, aparecen audioforos, videoforos, acertijos y chistes sexistas, que se convierten en detonantes para lograr sensibilizar a los/las asistentes al telecentro.

\section{Fase Ill: Cuestionar}

Preguntarse, indagar, querer conocer y aprender más, es lo que buscan los ejercicios realizados en dicha fase, para que los asistentes reflexionen sobre los procesos que realizan.
Las actividades o ejercicios lúdicos continúan, pero con situaciones reales o imaginarias, en las que los participantes deben generar estrategias y encontrar soluciones al problema que se les plantee. Las situaciones abarcan varios temas, entre los que están: las funciones de género, peleas por el poder, machismo, incomprensión, discriminación, falta de oportunidades, trabajo en equipo, entre otros.

"Lentes de género" es el tema central de dicha fase. Su apuesta es la de identificar los problemas de género y catalogarlos de acuerdo con su nivel de gravedad.

\section{Fase III: Proponer}

Esta es la fase que finaliza un proceso de reflexión. Por lo tanto, aquí se pretende fortalecerlo mediante la discusión y concertación de los temas principales del proceso: TIC, perspectiva de género, usos, prácticas y apropiación social. Se recuerdan conceptos, situaciones y las respectivas soluciones que generaron un cambio social.

Por otro lado y como complemento, se llega hasta el fondo con el modelo de autoevaluación, el cual permite identificar en la comunidad en qué nivel de procesos 
comunitarios están participando con indicadores e informes, sino con y cada uno de los conceptos tra- la elaboración detallada por mebajados durante el desarrollo del dio de la observación, de productos proyecto.

\section{Fase IV: Recolectar}

Entrevistas, grupos focales, encuestas, observación participante y valoraciones generales del proceso a todos los involucrados, sean hombres o mujeres, permiten recolectar la información suficiente para recopilar aspectos relevantes e identificar la manera como los participantes apropiaron el proceso... qué tanto modificó sus relaciones interpersonales y comunitarias, y sobre todo, identificar el grado de apropiación de las TIC.

\section{Fase V: Producir}

En esta fase se encuentra condensada la producción de tres crónicas, tres perfiles, una historia de vida, un fotorreportaje, videos cortos y productos elaborados por los asistentes a los talleres, que revelan el proceso realizado en los telecentros durante el proyecto "Implementación de la metodología GEM en torno a las TIC con perspectiva de género".

El objetivo de este punto consistió en mostrar un proceso, no solo que resaltaran las experiencias y frases más comunes de los participantes, aparte del cambio de perspectiva que generó para algunos.

\section{Consideraciones finales}

El reconocimiento que actualmente tienen las mujeres en todos aspectos o ámbitos catalogados en el Derecho Internacional Humanitario por sí solo resulta insuficiente para dar una respuesta adecuada a la diversidad, especificidad y complejidad de la desigualdad entre mujeres y hombres. La construcción de una sociedad realmente igualitaria implica necesariamente la adopción de instrumentos y el desarrollo de una serie de políticas puntuales que consideren la situación de vulnerabilidad que experimentan las mujeres por el solo hecho de serlo (PNUD, 2007).

En esta medida, entre las principales reflexiones que suscitó la implementación y adaptación de una guía de evaluación con perspectiva de género para usuarios/as de telecentros (GEM para telecentros) localizados en comunidades vulnerables o en condiciones prioritarias, se pueden destacar las siguientes desde el 
orden metodológico, de investigación, implementación y teórico.

- Abordar temas relacionados con la equidad de género, los derechos de mujeres y hombres, las oportunidades de participación; los las funciones productivas, reproductivas o comunitarias y su relación con las TIC, entres otras, no resultan ser temas considerados desde ningún punto de vista como prioritarios o relevantes en el desarrollo local de comunidades que, como las de Villapaz o Santa Elena, cuentan con fuertes creencias o valores tradicionales muy arraigados.

- Formar o consolidar un equipo de trabajo capaz de sortear cuestionamientos, reacciones o sentimientos que estos temas avivan en muchas de los/las participantes cuando comienzan a ser abordados, así como lograr un distanciamiento o una capacidad de análisis y crítica medianamente objetiva, se tornó en uno de los principales retos al momento de implementar la metodología.

- La convocatoria de hombres y personas adultas mayores, en particular, resulta desafiante durante el proceso. Para los primeros, aunque inquietos e interesados en un comienzo, con el transcurrir de las actividades no lograban identificarse plenamente con los temas tratados, se sentían "atacados" o no los encontraban importantes para sus vidas; y los segundos sentían que por su edad no debían ni siquiera acercarse al telecentro, eso se lo dejaban a los nietos y a los jóvenes de la zona.

- En cuanto al plan de trabajo, la ejecución de actividades y el cumplimiento de las metas, ya en "terreno", en el trabajo de campo en sí, las condiciones cambiaban considerablemente. El proceso no dependía únicamente de lo escrito en los talleres, su verdadera riqueza y esencia se encontraba en los y las participantes, quienes de un tema a otro podían tomarse más tiempo del previsto o deseaban profundizar, de lo contrario no consideraban pasar a una nueva etapa. Por tanto, manejar un límite de tiempo con los talleres y discusiones que actividad tras actividad se presentaban, era un desafío 
adicional. Se hacía necesario que el equipo de trabajo en terreno tuviese la experticia y capacidad de encaminar las discusiones, de no permitir que los y las participantes se perdieran en temas secundarios y no se llegara a conclusiones.

\section{Recomendaciones metodológicas para implementar la GEM en telecentros}

- Si bien es cierto que el material que recopila multimedia recoge cuidadosamente el paso a paso de la implementación de la metodología, es clave un acompañamiento del proceso con un/a facilitador/a capacitado/a en el tema (http://www.colombiadigital.net/universidad-autonomade-occidente/producciones/ multimediales/gem-una-herramienta-de-aprendizaje-para-elcambio-y-el-empoderamiento. html).

- El perfil, la calidad y el conocimiento del/la facilitador/a de la comunidad es clave porque en los talleres se manejan conceptos y discusiones sobre temas que generan polémica.

- Reconocimiento previo de la comunidad, sus líderes, voceros/as, quiénes son tomadores/as de decisiones, involucrarlos/as, invitarlos/as, hacerlos/as partícipes de todo el proceso.

- La metodología o manual GEM original se encuentra elaborada en un lenguaje formal y con un toque académico que no facilita la comprensión por parte del común de las personas; por tanto, es clave el estudio detallado y juicioso de este material previamente por parte del grupo, pero no es recomendable compartirlo exactamente igual con las comunidades, pues puede resultar muy confuso y desmotivar a los/ las participantes.

- La planeación previa de los talleres, la estructuración de actividades que generen algún tipo de acción en los/las participantes, resultan una muy buena estrategia para mantener la abtención y el interés de los/las participantes.

- Se diseñaron materiales, talleres, dinámicas, metodologías para orientar y aterrizar cada tema. Tener un instructivo para cada taller daba más seguridad a los/ las facilitadores/as.

- Los/as facilitadores/ras requieren te habilidades de conciliación para llamar al orden en los 
talleres cuando se presentan discusiones subidas de tono.

- En la etapa de recolección de información es recomendable contar con personal de apoyo extra, ello permite una mejor recolección de datos y sistematización de las experiencias.

Para finalizar vale pena destacar los principales alcances o logros que luego del proceso de sistematización del proyecto en el formato multimedial se han obtenido, entre estos:

- Las mujeres y los hombres que participaron en los talleres ganaron empoderamiento $\mathrm{y}$ autoestima. Ahora comentan que tienen relaciones más equitativas con sus esposos e hijos. Al final del proceso se sentían más capaces para trabajar en bien de la comunidad y participar en procesos sociales.

- El trabajo en grupo durante los talleres permitió que muchas personas perdieran el miedo a hablar en público, a expresar sus ideas sin temor a ser señalados e incluso a mejorar su autoestima.

- Se logró la participación de niños, niñas y adolescentes en los talleres. Esto es clave para inculcar valores de equidad y género en las nuevas generaciones.

- Muchas de las mujeres que participaron en el proceso están usando más las TIC y por medio de ellas buscan alternativas de ingresos, de educación, etcétera.

- Tanto las organizaciones coordinadoras o líderes del proceso, como aquellas que lo respaldaron, ganaron conocimiento $y$ apropiación en temas de género. Ahora muchas de ellas tienen en cuenta la metodología de evaluación para otras iniciativas en curso.

- Se adaptó y aprobó una metodología que fue implementada luego en veintidós nuevos telecentros Compartel, localizados en nueve departamentos de Colombia. Inciden a nivel práctico en el modelo de telecentros instalados por el Gobierno nacional.

- Se pasó de un equipo base formado en el tema (entre seis a ocho personas), a un grupo de más de veinte facilitadores/as que trabajaron la herramienta GEM en el ámbito nacional.

- El producto multimedial o multimedia GEM para telecentros fue compartido en el Encuentro 
de la Metodología GEM (celebrado en Santo Domingo, República Dominicana, en el 2009), con funcionarios/as y representantes de Guatemala, Costa Rica, Panamá, El Salvador y México.

- Asimismo, fue replicado en el Segundo GEM Global Training Exchange en Balí, Indonesia, del 24 al 30 de noviembre de 2009, en el que participaron representantes de países de África, Asia, Europa y América.

\section{Referencias}

Asociación para el Progreso de las Comunicaciones (APC) (2009). Carta de la APC sobre derechos en Internet. Recuperado de http://www.apc. org/es/node/5795.

Asociación para el Progreso de las Comunicaciones (APC) (2009). TIC para consolidar democracia con énfasis en el empoderamiento. Recuperado de http://www.apc.org/es/ pubs/books/tic-para-consolidar-democracia-con-enfasis-en-el-e.

Bernechea, M., González, E. \& Morgan, M. (2001). La sistematización como producción de conocimientos. Recuperado de http://preval.org/documentos/00508.pdf.
Cabrera, V. (2005). Apropiación social de la implementación comunitaria de TIC. Identidad, desarrollo y participación ciudadana en la experiencia de la red de información comunitaria de la Araucanía. Recuperado de http://www.flacsoandes.org/ dspace/handle/10469/121.

Castells, M. (2008). Tecnologías de la información, globalización y desarrollo social. En Antología de la comunicación para el cambio social: lecturas históricas y contemporáneas. La Paz: Plural.

Córdoba, R. (2007). Capacidades y libertad. Una aproximación a la teoría de Amartya Sen. Revista Internacional de Sociología (RIS), 47 47, 9-22.

Cumbre Mundial sobre la Sociedad de la Información. (Ginebra, 2003 Túnez, 2005). Declaración de Principios. Construir la Sociedad de la Información: un desafío global para el nuevo milenio. Recuperado de http://www.itu.int/wsis/docs/geneva/ official/dop-es.html.

De la Cruz, C. (1998). Guía metodológica para integrar la perspectiva de género en proyectos y programas de desarrollo. Recuperado de http://www. emakunde.euskadi.net/u72-publicac/es/contenidos/informacion/ 
pub_guias/es_emakunde/adjuntos/ guia_genero_es.pdf.

Del Río, O. (2009). TIC, derechos humanos y desarrollo: nuevos escenarios de la comunicación social, 55-69. Recuperado de ddd.uab.cat/ pub/analisi/02112175n38p55.pdf.

Finquelievich, S. \& Lago S. (2004). Mujeres en América Latina y el Caribe: ¿son las tecnologías de información y comunicación un arma efectiva para luchar contra la pobreza? Revista venezolana de estudios de la mujer, 9 22, 129-144.

Gurumurthy, A. (2004). Género y TIC. Informe General. Bridge Institute of Development Studies. Recuperado de: http://www.bridge.ids. ac.uk/reports/cep-icts-or.pdf

Hafkin, N. \& Huyer, S. (2007). Women and Gender in ICT Statistics. Recuperado de http://itidjournal. org/itid/article/view/254/124.

Hafkin, N. \& Taggart, N. (2009). El género, la tecnología de la información y los países en desarrollo. Estudio analítico. Academy for Educational Development (AED) for the Office of Women in Development, Bureau for Global Programs, Field Support and Research, US Agency for International Development (USAID). Recuperado de http://learnlink.aed.org/Publications/espanol/ El_G\%E9nero_Resumen_Ejecutivo. pdf.

Programa de las Naciones Unidas para el Desarrollo (PNUD)(2001). TIC: una mirada desde la perspectiva de género. Informe Nacional sobre Desarrollo Humano Ecuador 2001: las tecnologías de la información y comunicación para el desarrollo humano. Recuperado de http://78.46.95.73:8080/jspui/handle/ 123456789/725.

Programa de las Naciones Unidas para el Desarrollo (PNUD). Escuela virtual PNUD. Curso acceso y aplicaciones de las TIC para el desarrollo humano. Módulo 4: Políticas TIC y desarrollo humano. Notas del profesor. Recuperado de http:// escuelapnud.org/biblioteca/pmb/ opac_css/index.php?lvl=more results\&mode=keyword\&user_ query=TIC.

Rueda, R. (2005). Apropiación social de las tecnologías de la información: Ciberciudadanías emergentes. Recuperado de http://alainet.org/active/9896. 
Ruiz, L. (2001). La sistematización de ber 2003. Recuperado de http://www. prácticas. Recuperado de http://www. un.org/apps/sg/sgstats.asp?nid=695. oei.es/equidad/liceo.PDF.

Teltscher, S. (2002). Gender, ICT Sabanes, D. (2004). Mujeres y nueand Development. United Nations vas tecnologías de la información Conference on Trade and Developy la comunicación. Recuperado de ment (UNCTAD). Recuperado de http://www.cuadernos.tpdh.org/ file_upload/02_Dafne_Sabane.pdf.

Secretary-General's address to the www.worldcivilsociety.org/documents/17.13_teltscher_susanne_ unctad.doc

World Summit on the Information Society. Geneva, Switzerland, 10 Decem- 